\title{
Logarithmic corrections to entropy for black holes with hyperbolic horizon
}

\author{
Yun Soo Myung 1 \\ Institute of Basic Science and School of Computer Aided Science \\ Inje University, Gimhae 621-749, Korea
}

\begin{abstract}
We compute logarithmic corrections to entropy for black holes with hyperbolic horizon. For this purpose, we introduce the topological black hole and MTZ black holes in four dimensions, while in five dimensions, the topological black holes and Gauss-Bonnet black hole with negative coupling are considered. As they stand, logarithmic corrections are problematic because small black holes with positive heat capacity have negative energies. However, introducing the background state of extremal black hole, the logarithmic corrections are performed for black hole with hyperbolic horizon.
\end{abstract}

\footnotetext{
${ }^{1}$ e-mail address: ysmyung@inje.ac.kr
} 


\section{Introduction}

A number of authors have shown that for a large class of black holes, the BekensteinHawking entropy receives logarithmic corrections due to thermodynamic fluctuations around thermal equilibrium [1, 2]. Up to now, a corrected-entropy formula takes the form

$$
S_{c}=S-\frac{1}{2} \ln \left[C T^{2}\right]+\cdots,
$$

where $C$ and $T$ are the heat capacity and temperature of the given black hole, and $S$ denotes the uncorrected Bekenstein-Hawking entropy. Here $C$ should be positive for Eq.(1) to be well-defined. We note that for $C>0(C<0)$, the system is thermodynamically stable (unstable). A black hole with negative specific heat is in an unstable equilibrium with the heat reservoir of the temperature $T$ [3]. Its fate under small fluctuations will be either to decay to hot flat space or to grow without limit by emitting or absorbing thermal radiation in the heat reservoir [4]. Hence it is meaningless to apply Eq.(1) to the black hole with $C<0$. There exists a way to achieve a stable black hole in an equilibrium with the heat reservoir. A black hole could be rendered thermodynamically stable by placing it in AdS spacetimes. An important point is to understand how a black hole with positive specific heat could emerge from thermal radiation through a phase transition. To this end, one introduces the Hawking-Page phase transition between thermal AdS space and Schwarzschild-AdS black hole [5, 6, 7].

It is well known that the entropy-corrected formula of Eq.(1) is universal [2], implying that this prescription could apply to all black holes with positive specific heat. We note that the canonical ensemble was used to derive Eq.(1). In this case, the positive energy (mass) of the system is needed to define the canonical ensemble. However, small black holes with hyperbolic horizon have the negative energy, even though they have positive heat capacity. These may be counter examples, because they have negative masses in the range $M_{e} \leq M \leq 0$ with $M_{e}$ mass of extremal black hole. Hence, a direct application of Eq.(1) to these black holes is not guaranteed for accounting their thermodynamic fluctuations.

In this work, we address this issue and resolve this by introducing the background state of extremal black hole, similar to the charged black holes with spherical horizon [8]. The difference is that for hyperbolic horizon, its background state energy is negative, whereas its background state energy is positive for spherical horizon. In order to avoid negative mass, we use the substraction scheme. The entropy-corrected formula is not changed because the substraction scheme corresponds to a constant shifting from $M$ to $E=M-M_{e}$. We will use Eq.(1) to study thermal fluctuations of black holes with 
hyperbolic horizon.

The organization of this work is as follows. Section 2 is devoted to performing the logarithmic corrections to topological black hole (4DTBH) and MTZ black holes in four dimensions. We study the logarithmic corrections to topological black hole (5DTBH) and Gauss-Bonnet black holes in five dimensions in section 3. We discuss the AdS/CFT correspondences for these black hole in section 4 . Finally, we discuss our results in section 5.

\section{$2 \quad 4 \mathrm{DTBH}$ and MTZ black holes}

Topological black holes in asymptotically anti-de Sitter spacetimes were first found in three and four dimensions [9]. Their black hole horizons are Einstein spaces of spherical $(k=1)$, hyperbolic $(k=-1)$, and flat $(k=0)$ curvature for higher dimensions more than three [10, 11]. The standard equilibrium and off-equilibrium thermodynamic analyses are possible to show that they are treated as the extended thermodynamic systems, even though their horizons are not spherical. The topological black holes in four-dimensional AdS spacetimes are given by

$$
d s_{4 D T B H}^{2}=g_{\mu \nu} d x^{\mu} d x^{\nu}=-f_{T}(r) d t^{2}+\frac{1}{f_{T}(r)} d r^{2}+r^{2} d \Sigma_{k}^{2},
$$

where the metric function $f_{T}(r)$ is given by

$$
f_{T}(r)=k-\frac{m}{r}+\frac{r^{2}}{l^{2}}
$$

$d \Sigma_{k}^{2}$ describes the $2 \mathrm{D}$ horizon geometry with a constant curvature

$$
d \Sigma_{k}^{2}=d \theta^{2}+f_{k}^{2}(\theta) d \phi^{2}
$$

where $f_{k}(\theta)$ is given by

$$
f_{0}(\theta)=\theta, f_{1}(\theta)=\sin \theta, f_{-1}(\theta)=\sinh \theta .
$$

Here we define $k=1,0$, and -1 cases as the 4D Schwarzschild-AdS black hole (4DSAdS), 4D flat-AdS black hole, and 4D hyperbolic-AdS black hole (=4DTBH) [12, respectively. In the case of $k=1, m=0$, we have an $\mathrm{AdS}_{4}$-spacetime with its curvature radius $l$. However, $m \neq 0$ generates the topological black holes. It is easy to check that the metric (2) satisfies Einstein's equations with a negative cosmological constant

$$
R_{\mu \nu}=-\frac{3}{l^{2}} g_{\mu \nu}
$$


when the horizon is an Einstein space

$$
R_{i j}=k h_{i j}
$$

In this work, we are interested in the negative curvature with $k=-1$ only. Then, the horizon space is a hyperbolic manifold of $\Sigma_{k=-1}=H^{2} / \Gamma$, where $H^{2}$ is $2 \mathrm{D}$ hyperbolic space and $\Gamma$ is a suitable discrete subgroup of the isometry group of $H^{2}$ [13].

First of all, the 4DTBH provides thermodynamic quantities of Hawking temperature $T_{T}$, mass $M_{T}$, entropy $S_{T}$, heat capacity $C_{T}$, and free energy $F_{T}=M_{T}-T_{T} S_{T}$

$$
\begin{aligned}
& T_{T}=\frac{1}{4 \pi \rho_{+}}\left(\frac{3 \rho_{+}^{2}}{l^{2}}-1\right), M_{T} \equiv \frac{\sigma}{8 \pi G_{4}} m=\frac{\sigma \rho_{+}}{8 \pi G_{4}}\left(\frac{\rho_{+}^{2}}{l^{2}}-1\right), \\
& S_{T}=\frac{\sigma \rho_{+}^{2}}{4 G_{4}}, C_{T}=2\left[\frac{3 \rho_{+}^{2}-l^{2}}{3 \rho_{+}^{2}+l^{2}}\right] S_{T}, F_{T}\left(\rho_{+}\right)=-\frac{\sigma \rho_{+}}{16 \pi G_{4}}\left(\frac{\rho_{+}^{2}}{l^{2}}+1\right),
\end{aligned}
$$

where $\sigma$ denotes the area of a unit 2D hyperbolic space $\Sigma_{k=-1}$ and $\rho_{+}$is the outer horizon which satisfies $f_{T}=0$ with $k=-1$. We note that the first-law of thermodynamics $d M_{T}=T_{T} d S_{T}$ holds for the $4 \mathrm{DTBH}$. Also we observe that the entropy satisfies the area-law.

Importantly, we observe that the temperature and heat capacity are positive for $\rho_{+}>$ $\rho_{e}=l / \sqrt{3}$, while the mass is positive only for $\rho_{+}>l$. This means that any canonical ensemble is not defined for $\rho_{+}<l$. Thus it seems that the entropy-correction formula of Eq.(1) is useless for describing thermal fluctuations of the small 4DTBH. This arises mainly because its horizon geometry is hyperbolic.

In order to resolve this problem, we have to choose an appropriate substraction scheme. In the Reissner-Norström-AdS black hole, one has introduced the extremal black hole as the background state to define an appropriate free energy in the canonical ensemble [8]. Similarly, we wish to introduce the extremal black hole at $\rho_{+}=\rho_{e}$ as the background state even though the charge is absent in the $4 \mathrm{DTBH}$ [14]. In this case, we define the positive energy as

$$
E_{T}=M_{T}-M_{T}^{e}
$$

with respect to the negative background energy

$$
M_{T}^{e}=\left.M_{T}\right|_{\rho_{+}=\frac{l}{\sqrt{3}}}=-\frac{\sigma l}{12 \sqrt{3} \pi G_{4}} .
$$

Although one uses $E_{T}$ instead of $M_{T}$, all thermodynamics except $F_{T}$ remain unchanged. The free energy is an important quantity to discuss the global stability and phase transition. In this case, it is shifted by $F_{M}^{n}=E_{T}-T_{M} S_{M}$. Then, for $\rho_{+}>\rho_{e}$, we may use the 
corrected-entropy formula to study thermodynamic fluctuations around the equilibrium $4 \mathrm{DTBH}$. We prove it by showing that the root-mean-square energy fluctuations defined by the square root of

$$
<\left(\Delta E_{T}\right)^{2}>=C_{T} T_{T}^{2}
$$

remains unchanged under the change from $M_{T}$ to $E_{T}$. In deriving Eq.(11), one has used $S_{c}=S-\frac{1}{2} \ln S^{\prime \prime}+\cdots$ with $S^{\prime \prime}=<M^{2}>-<M>^{2}=C T^{2}$. It is easy to check $<E^{2}>-<E>^{2}=<M^{2}>-<M>^{2}$ under the shifting $M \rightarrow E=M-M_{e}$, which is also confirmed by noting that $C T^{2}$ is an invariant quantity. Then, the mass (energy) becomes positive and thus one could define the canonical ensemble to discuss its thermal fluctuations around the equilibrium configuration. Importantly, the entropy-corrected formula is not changed because the substraction scheme corresponds to a constant shifting from $M$ to $E$.

For a large $4 \mathrm{DTBH}$ with $\rho_{+} \gg l$, we have approximate forms

$$
C_{T} \sim 2 S_{T}, T_{T}^{2} \sim S_{T}
$$

which leads to an approximately correct-entropy formula

$$
S_{T}^{c} \sim S_{T}-\ln \left[S_{T}\right]+\cdots
$$

This is the same form as for large 4DSAdS [2]. Furthermore, we note that the Smarr formula of $M=T S / 2$ (Euler relation) is not satisfied for the choice of either $M=M_{T}$ or $E_{T}$.

On the other hand, the MTZ black hole dressed by scalar could be obtained from the Einstein action minimally coupled to a scalar in AdS spacetimes [15]

$$
I_{4}[g, \phi]=\int d^{4} x \sqrt{-g}\left[\frac{R-2 \Lambda_{4}}{16 \pi G_{4}}-\frac{1}{2}(\nabla \phi)^{2}-V(\phi)\right]
$$

where the potential $V(\phi)$ is given by

$$
V(\phi)=-\frac{3}{4 \pi G_{4} l^{2}} \sinh ^{2}\left[\sqrt{\frac{4 \pi G_{4}}{3}} \phi\right] .
$$

Here $\Lambda_{4}=-3 / l^{2}$ with $l$ the curvature radius of $\mathrm{AdS}_{4}$ spacetimes. For $\phi=0$ case, we obtain the action for the $4 \mathrm{DTBH}$. In order to understand the role of scalar and its potential, it would be better to go the conformal frame by introducing a conformal factor $\Psi=\sqrt{3 / 4 \pi G_{4}} \tanh \left[\sqrt{4 \pi G_{4} / 3} \phi\right]$. Performing a conformal transformation as $\hat{g}_{\mu \nu}=(1-$ $\left.4 \pi G \Psi^{2} / 3\right)^{-1} g_{\mu \nu}$, the action $I_{4}$ takes the from

$$
I_{4}[\hat{g}, \Psi]=\int d^{4} x \sqrt{-\hat{g}}\left[\frac{\hat{R}-2 \Lambda_{4}}{16 \pi G_{4}}-\frac{1}{2}(\nabla \Psi)^{2}-\frac{1}{12} \hat{R} \Psi^{2}-\frac{2 \pi G_{4}}{3 l^{2}} \Psi^{4}\right],
$$


where a conformally coupled scalar $\Psi$ appears with the conventional potential $\Psi^{4}$. In connection with higher derivative terms, this potential may play a role of curvaturesquared term $\hat{R}^{2}$. It provides a black hole dressed by scalar with hyperbolic horizon. On the other hand, for $\Psi=0$, one has the action for 4DTBH, Eq.(2) . Then, the solution of the MTZ black hole is given by

$$
d s_{M}^{2}=-f_{M}(r) d t^{2}+\frac{d r^{2}}{f_{M}(r)}+r^{2} d \Sigma_{k=-1}^{2}
$$

where the metric function $f_{M}(r)$ is given by

$$
f_{M}(r)=\frac{r^{2}}{l^{2}}-\left(1+\frac{G_{4} \mu}{r}\right)^{2}
$$

and a conformally coupled scalar has the configuration

$$
\bar{\Psi}(r)=\sqrt{\frac{3}{4 \pi G_{4}}} \frac{G_{4} \mu}{r+G_{4} \mu} .
$$

Thermodynamic quantities of the MTZ are given by Hawking temperature $T_{M}$, mass $M_{M}$, entropy $S_{M}$, heat capacity $C_{M}$, and free energy $F_{M}=M_{M}-T_{M} S_{M}$ by [15, 16, 17]

$$
\begin{aligned}
& T_{M}=\frac{1}{2 \pi l}\left[\frac{2 r_{+}}{l}-1\right]=\left(\frac{2 G_{3}}{\sigma \pi l^{3}}\right) S_{M}, M_{M} \equiv \frac{\sigma}{4 \pi} \mu=\frac{\sigma r_{+}}{4 \pi G_{4}}\left(\frac{r_{+}}{l}-1\right), \\
& S_{M}=\frac{\sigma l^{2}}{4 G_{4}}\left(\frac{2 r_{+}}{l}-1\right)=C_{M}, F_{M}\left(r_{+}\right)=-\frac{\sigma}{8 \pi G_{4}}\left(\frac{2 r_{+}^{2}}{l}-2 r_{+}+l\right),
\end{aligned}
$$

where $r_{+}$is the outer horizon which satisfies $f_{M}=0$. We note that the first-law of thermodynamics $d M_{M}=T_{M} d S_{M}$ is satisfied for the MTZ. Also we observe that the entropy $S_{M}$ was obtained by using the Wald's formula but it does not satisfy the arealaw. Importantly, the temperature and heat capacity are positive for $r_{+}>r_{e}=l / 2$, while the mass is positive for $r_{+}>l$ only. This means that any canonical ensemble is not suitable for describing the case of $r_{+}<l$ and thus the corrected-entropy formula of Eq.(1) may be useless for the small MTZ black hole.

In order to resolve this problem, we have to choose an appropriate background state. Similarly, we introduce the extremal black hole as the background state even though the charge is absent in the MTZ black hole, too. For the MTZ black hole, we define the positive energy as [18]

$$
E_{M}=M_{M}-M_{M}^{e}
$$

with the background state energy

$$
M_{M}^{e}=\left.M_{M}\right|_{r_{+}=\frac{l}{2}}=-\frac{\sigma l}{16 \pi G_{4}} .
$$


Although one uses $E_{M}$ instead of $M_{M}$, all thermodynamics of the MTZ except $F_{M}^{n}=$ $E_{M}-T_{M} S_{M}$ remain unchanged. Nicely, we check that the Smarr formula

$$
E_{M}=\frac{T_{M} S_{M}}{2}
$$

is satisfied for $r_{+}>r_{e}$ only when using the positive energy $E_{M}$. This implies that we could use the corrected-entropy formula to see thermodynamic fluctuations around the equilibrium MTZ, even though its entropy does not satisfy the area-law. In this case, we have a corrected-entropy formula for $r_{+}>r_{e}$

$$
S_{M}^{c}=S_{M}-\frac{3}{2} \ln \left[S_{M}\right]+\cdots
$$

which is the same formula for the non-rotating BTZ black hole [2] whose thermodynamic quantities are given by

$$
T_{B}=\frac{r_{+}}{2 \pi l^{2}}=\left[\frac{G_{3}}{\pi^{2} l^{2}}\right] S_{B}, M_{B}=\frac{r_{+}^{2}}{8 G_{3} l^{2}}=E_{B}, S_{B}=\frac{\pi r_{+}}{2 G_{3}}=C_{B}, F_{B}=-\frac{r_{+}^{2}}{8 G_{3} l^{2}} .
$$

Here we have the Smarr formula of $E_{B}=T_{B} S_{B} / 2$ for the non-rotating BTZ black hole. Two are very similar in the sense that they satisfy the Smarr formula and have the same corrected-entropy formula. A difference is that these formulae are valid for the outer horizon $r_{+}>r_{e}$ of MTZ black hole and for any size of non-rotating BTZ black hole. Furthermore, we derive thermodynamic quantities for large MTZ black hole with $r_{+} \gg l$ as

$$
T_{M} \simeq \frac{r_{+}}{\pi l^{2}}, M_{M} \simeq \frac{\sigma r_{+}^{2}}{4 \pi l G_{4}}, S_{M}=C_{M} \simeq \frac{\sigma l r_{+}}{2 G_{4}}, F_{M} \simeq-\frac{\sigma r_{+}^{2}}{4 \pi l G_{4}}
$$

which show the nearly same behavior as Eq. (26) is shown. Particularly, choosing $\sigma=\pi / l$ leads to close relations of $T_{M}=2 T_{B}, M_{M}=2 M_{B}, F_{M}=2 F_{B}, S_{M}=S_{B}, C_{M}=C_{B}$. This suggests that the MTZ black hole dressed by scalar is a corner stone for the $4 \mathrm{D}$ black hole physics as the non-rotating BTZ black hole does play a key role in the 3D black hole physics.

\section{$3 \quad$ 5DTBH and Gauss-Bonnet black holes}

In five dimensions, the topological AdS black holes are given by

$$
d s_{5 D T B H}^{2}=-h(r) d t^{2}+\frac{1}{h(r)} d r^{2}+r^{2} d \Sigma_{k}^{2},
$$


where $d \Sigma_{k}^{2}=d \chi^{2}+h_{k}^{2}(\chi)\left(d \theta^{2}+\sin ^{2} \theta d \phi^{2}\right)$ describes the 3D horizon geometry with a constant curvature. Further $h(r)$ and $h_{k}(\chi)$ are given by

$$
h(r)=k-\frac{m}{r^{2}}+\frac{r^{2}}{\ell^{2}}, \quad h_{0}(\chi)=\chi, h_{1}(\chi)=\sin \chi, h_{-1}(\chi)=\sinh \chi .
$$

Here we define $k=1,0$, and -1 cases as the 5D Schwarzschild-AdS black hole (5DSAdS) [19, 20], 5D flat-AdS black hole, and 5D hyperbolic-AdS black hole (5DTBH) [21], respectively. We are interested in the $k=-1$ case only. In this case, the location of the event horizon is given by

$$
r_{+}^{2}=\frac{\ell^{2}}{2}\left(1+\sqrt{1+4 m / \ell^{2}}\right) .
$$

The relevant thermodynamic quantities of Hawking temperature $T_{t}$, mass $M_{t}$, BekensteinHawking entropy $S_{t}$, heat capacity $C_{t}$, and free energy $F_{t}=M_{t}-T_{t} S_{t}$ are given by

$$
\begin{aligned}
& T_{t}=\frac{1}{2 \pi r_{+}}\left(\frac{2 r_{+}^{2}}{\ell^{2}}-1\right), M_{t} \equiv \frac{3 V_{3} m}{16 \pi G_{5}}=\frac{3 V_{3} r_{+}^{2}}{16 \pi G_{5}}\left(\frac{r_{+}^{2}}{l^{2}}-1\right), \\
& S_{t}=\frac{V_{3} r_{+}^{3}}{4 G_{5}}, C_{t}=3\left[\frac{2 r_{+}^{2}-\ell^{2}}{2 r_{+}^{2}+\ell^{2}}\right] S_{t}, F=-\frac{V_{3} r_{+}^{2}}{16 \pi G_{5}}\left(\frac{r_{+}^{2}}{\ell^{2}}+1\right)
\end{aligned}
$$

where $V_{3}$ is the volume of a unit $3 \mathrm{D}$ hyperbolic space $\Sigma_{k=-1}$ and $G_{5}$ is the five-dimensional Newton constant. Here we find that $T_{t}, C_{t}>0$ for $r_{+}>r_{e}=l / \sqrt{2}$, while $M_{t}>0$ for $r_{+}>l$. It seems that any canonical ensemble is not defined for $r_{+}<l$ and thus the entropy-correction formula of Eq.(11) is meaningless for the small $5 \mathrm{DTBH}$.

In order to cure this problem, we have to find an appropriate substraction scheme. Similarly, we introduce the extremal black hole as the background state. In this case, we define the positive energy as

$$
E_{t}=M_{t}-M_{t}^{e}
$$

with the background state energy

$$
M_{t}^{e}=\left.M_{t}\right|_{r_{+}=\frac{l}{\sqrt{2}}}=-\frac{3 V_{3} l^{2}}{64 \pi G_{5}} .
$$

Although one uses $E_{t}$ instead of $M_{t}$, all thermodynamics except $F_{t}^{n}=E_{t}-T_{t} S_{t}$ remain unchanged. This implies that for $r_{+}>r_{e}$, we could use the corrected-entropy formula to study thermodynamic fluctuations around the equilibrium 5DTBH.

For a large 5DTBH with $r_{+} \gg l$, we have approximate forms

$$
C_{t} \sim 3 S_{t}, T_{t}^{2} \sim S_{t}^{\frac{2}{3}}
$$

which leads to an approximately correct-entropy formula

$$
S_{t}^{c} \sim S_{t}-\frac{5}{6} \ln \left[S_{t}\right]+\cdots .
$$


This is the same form as for large 5DSAdS [2, 22]. Furthermore, we note that the Smarr formula is not satisfied for the choice of either $M=M_{t}$ or $E_{t}$.

In order to find a 5D version of MTZ black hole, we may consider the $5 \mathrm{D}$ action like Eq.(17) with replacing the last two terms and $\Lambda_{4}$ by $(3 / 32) R \Psi^{2}+\alpha_{5} \Psi^{10 / 3}$ and $\Lambda_{5}$. However, it was turned out that there is no solution like the MTZ black hole in $\mathrm{AdS}_{5}$ spacetimes [18]. That is, there is no such a 5D black hole dressed by scalar. Instead, we consider a similar black hole with hyperbolic horizon. This may be found from a 5D gravitational action in the presence of a negative cosmological constant $\Lambda_{5}=-6 / l^{2}$ and Gauss-Bonnet (GB) term as

$$
I_{5}[g, c]=\frac{1}{16 \pi G_{5}} \int d^{5} x \sqrt{-g}\left[R-2 \Lambda_{5}+\frac{c}{2} L_{G B}\right],
$$

where

$$
L_{G B}=R^{2}-4 R_{\mu \nu} R^{\mu \nu}+R_{\mu \nu \rho \sigma} R^{\mu \nu \rho \sigma} .
$$

Here $c$ is a GB coupling constant having mass dimension -2 . For $c=0$, this action reduces to the action for the 5DTBH, Eq.(28). The solution of the TGBAdS black hole is given by

$$
d s_{g}^{2}=-f_{g}(r) d t^{2}+\frac{d r^{2}}{f_{g}(r)}+r^{2} d \Sigma_{k}^{2}
$$

The metric function is given by

$$
f_{g}(r)=k+\frac{r^{2}}{2 c}\left[1+\epsilon \sqrt{1+\frac{4 c}{3}\left(\frac{2 \mu}{r^{4}}-\frac{3}{l^{2}}\right)}\right] .
$$

Hereafter, we consider the negative coupling of $c<0$ with $k=-1$ and $\epsilon=-1$ in accordance with the hyperbolic horizon.

For the TGBAdS black hole, we have relevant thermodynamic quantities [23, 24, 25]

$$
\begin{aligned}
& T_{g}=\frac{r_{+}}{2 \pi\left(r_{+}^{2}-2 c\right)}\left(\frac{2 r_{+}^{2}}{l^{2}}-1\right), M_{g} \equiv \frac{V_{3} \mu}{8 \pi G_{5}}=\frac{3 V_{3} r_{+}^{2}}{16 \pi G_{5}}\left(\frac{r_{+}^{2}}{l^{2}}-1+\frac{c}{r_{+}^{2}}\right), \\
& S_{g}=\frac{V_{3} r_{+}^{3}}{4 G_{5}}\left(1-\frac{6 c}{r_{+}^{2}}\right), C_{g}=3 \frac{V_{3} r_{+}^{3}}{4 G_{5}}\left[\frac{\left(2 r_{+}^{2}-l^{2}\right)\left(1-2 c / r_{+}^{2}\right)^{2}}{\left(2 r_{+}^{2}+l^{2}\right)-\left(2 c / r_{+}^{2}\right)\left(6 r_{+}^{2}-l^{2}\right)}\right], \\
& F_{g}=M_{g}-T_{g} S_{g}=-\frac{V_{3} r_{+}^{4}}{16 \pi G_{5}\left(r_{+}^{2}-2 c\right)}\left[\left(\frac{r_{+}^{2}}{l^{2}}+1\right)-\frac{3 c}{r_{+}^{4}}\left(\frac{6 r_{+}^{4}}{l^{2}}-r_{+}^{2}-2 c\right)\right],
\end{aligned}
$$

where we point out that $S_{g}$ was derived using the first-law of thermodynamics and thus it does not satisfy the area-law. Here we find that $T_{g}, C_{g}>0$ for $r_{+}>r_{e}=l / \sqrt{2}$. On the other hand, one has $M_{t}>0$ for $r_{+}>r_{0}$ where

$$
r_{0}=\frac{l}{\sqrt{2}} \sqrt{1+\sqrt{1-4 c / l^{2}}}>l
$$


which is determined by the massless condition of $M_{g}=0$. This means that any canonical ensemble is not defined for $r_{+}<r_{0}$ and thus the entropy-correction formula of Eq.(11) is useless for the small TGBAdS. In order to solve this problem, we have to find an appropriate background state. We introduce the extremal black hole as the background state. In this case, we define the positive energy as

$$
E_{g}=M_{g}-M_{g}^{e}
$$

with the background state energy

$$
M_{g}^{e}=\left.M_{g}\right|_{r_{+}=\frac{l}{\sqrt{2}}}=-\frac{3 V_{3}}{16 \pi G_{5}}\left[\frac{l^{2}}{4}-c\right] .
$$

At this stage, we find an important equality for energies

$$
E_{g}=E_{t}=\frac{3 V_{3} r_{+}^{2}}{16 \pi G_{5}}\left[\frac{r_{+}^{2}}{l^{2}}-1+\frac{l^{2}}{4 r_{+}^{2}}\right] .
$$

Although one uses $E_{g}$ instead of $M_{g}$, all thermodynamics except $F_{g}^{n}=E_{g}-T_{g} S_{g}$ remain unchanged. This implies that for $r_{+}>r_{e}$, we could use the corrected-entropy formula to see thermodynamic fluctuations around the equilibrium TGBAdS. For a large TGBAdS with $r_{+} \gg l, c$, we have approximate forms

$$
C_{g} \sim 3 S_{g}, T_{g}^{2} \sim S_{g}^{\frac{2}{3}}
$$

which leads to an approximately correct-entropy formula

$$
S_{g}^{c} \sim S_{g}-\frac{5}{6} \ln \left[S_{g}\right]+\cdots .
$$

This is the same form as for large 5DSAdS [2]. Furthermore, we note that the Smarr formula is not satisfied for the choice of either $M=M_{g}$ or $E_{g}$.

\section{Boundary conformal field theories}

The holographic principle means that the number of degrees of freedom associated with the bulk gravitational dynamics is determined by its boundary spacetime. We start with the bulk-relation between the entropy and energy for the non-rotating BTZ black hole

$$
S_{B}=\pi l \sqrt{\frac{2 E_{B}}{G_{3}}}
$$

which is derived using $E_{B}=\frac{\left(\pi l T_{B}\right)^{2}}{2 G_{3}}$ and Smarr formula of $E_{B}=\frac{T_{B} S_{B}}{2}$. 
Also this could be recovered from the CFT on the boundary at infinity using the Cardy formula

$$
S_{C F T}=2 \pi \sqrt{\frac{c L_{0}}{6}}+2 \pi \sqrt{\frac{\bar{c} \bar{L}_{0}}{6}}
$$

with

$$
c=\bar{c}=\frac{3 l}{2 G_{3}}, L_{0}=\bar{L}_{0}=\frac{E_{B} l}{2} .
$$

This means that the $\mathrm{AdS}_{3} / \mathrm{CFT}_{2}$ correspondence was realized for counting the BTZ black hole entropy.

However, as far as we know, there is no definite realization of the $\mathrm{AdS}_{4} / \mathrm{CFT}_{3}$ correspondence. Hence, we may try to understand this correspondence by analogy of the non-rotating BTZ black hole. First of all, we find the similar relation from $E_{M}=\frac{\sigma \pi l^{3} T_{M}^{2}}{4 G_{4}}$ and the Smarr formula of Eq.(24) as

$$
S_{M}=l \sqrt{\frac{\pi l \sigma E_{M}}{G_{4}}}=2 \pi l \sqrt{\left(-4 M_{M}^{e}\right) E_{M}}
$$

which is valid for the outer horizon $r_{+}>r_{e}$. Assuming that the Cardy formula

$$
S_{C F T}^{M}=2 \pi l \sqrt{\frac{c_{3} L_{0}^{M}}{6}}+2 \pi l \sqrt{\frac{\bar{c}_{3} \bar{L}_{0}^{M}}{6}}
$$

with

$$
c_{3}=\bar{c}_{3}=\frac{3 \sigma l}{2 \pi G_{4}}=-24 M_{M}^{e}, L_{0}^{M}=\bar{L}_{0}^{M}=\frac{E_{M}}{2}
$$

holds in three dimensions, we may recover the entropy $S_{M}$ from the boundary $\mathrm{CFT}_{3}$ entropy $S_{C F T}^{M}$. We note that Eqs.(51) and (52) are just our proposition. This is because we do not know what is the exact Cardy formula for the boundary $\mathrm{CFT}_{3}$. One thing to show is that one may recover the bulk entropy $S_{M}$ from the presumed formula (51) with (52).

The $\mathrm{AdS}_{5} / \mathrm{CFT}_{4}$ correspondence represents also a concrete realization of the holographic principle. In this case, there is no Smarr formula to derive a direct relation like Eqs. (47) and (50). Instead, one proposes the Cardy-Verlinde formula for a strongly coupled $\mathrm{CFT}_{4}$ with its AdS dual [26]. It is known that this formula holds for various kinds of asymptotically AdS spacetimes including the TAdS black holes [21]. The boundary spacetimes for the $\mathrm{CFT}_{4}$ are defined through the $\mathrm{AdS}_{5} / \mathrm{CFT}_{4}$ correspondence [27]

$$
d s_{C F T_{4}}^{2}=\lim _{r \rightarrow \infty} \frac{R^{2}}{r^{2}} d s_{5 D T B H}^{2}=-\frac{R^{2}}{l^{2}} d t^{2}+R^{2} d \Sigma_{k}^{2} .
$$

From the above, the relation between the five-dimensional bulk and four-dimensional boundary quantities is given by $E_{C F T}=(l / R) E$ and $T_{C F T}=(l / R) T$ where the size of 
boundary space $R$ satisfies $T_{C F T}>1 / R$. As is expected, we obtain the same entropy: $S_{C F T}=S$. We note that the boundary system at high temperature is described by the CFT-radiation with equation of state $p=E_{C F T} / 3 V_{3}$. Then, the Casimir energy defined by $E_{c}=3\left(E_{C F T}+p V_{3}-T_{C F T} S_{C F T}\right)$ [28] is necessary to obtain the Cardy-Verlinde formula

$$
S_{C F T}=\frac{2 \pi R}{3 \sqrt{|k|}} \sqrt{E_{c}\left(2 E_{C F T}-E_{c}\right)} .
$$

The non-zero Casimir energy reflects that the Euler relation is not satisfied. We find the boundary thermal quantities for 5DTBH as functions of $\hat{r}=r_{+} / l$ [12, 22, 29]

$$
\begin{aligned}
& E_{C F T}^{t}=\frac{3 V_{3} \kappa \hat{r}^{2}}{R}\left[\hat{r}^{2}-1+\frac{1}{4 \hat{r}^{2}}\right], \quad T_{C F T}^{t}=\frac{\hat{r}}{2 \pi R}\left[2 \hat{r}^{2}-1\right], \\
& E_{c}^{t}=-\frac{3 V_{3} \kappa \hat{r}^{2}\left(2 \hat{r}^{2}-1\right)}{R}
\end{aligned}
$$

with $\kappa=l^{3} / 16 \pi G_{5}$. On the other hand, for the TGBAdS case, we find the boundary thermal quantities

$$
\begin{aligned}
& E_{C F T}^{g}=\frac{3 V_{3} \kappa \hat{r}^{2}}{R}\left[\hat{r}^{2}-1+\frac{1}{4 \hat{r}^{2}}\right], \quad T_{C F T}^{g}=\frac{\hat{r}}{2 \pi R\left(\hat{r}^{2}-2 \hat{c}\right)}\left[2 \hat{r}^{2}-1\right] \\
& E_{c}^{g}=-\frac{3 V_{3} \kappa \hat{r}^{2}\left(2 \hat{r}^{2}-1\right)}{R}\left[\frac{(1-8 \hat{c}) \hat{r}^{2}-2 \hat{c}}{\hat{r}^{2}-2 \hat{c}}\right] .
\end{aligned}
$$

Concerning the AdS/CFT correspondence, we remind the reader that the boundary $\mathrm{CFT}_{4}$ energy $\left(E_{C F T}\right)$ should be positive in order for it to make sense. Also the Casimir energy $\left(E_{c}\right)$ is related to the central charge of the corresponding $\mathrm{CFT}_{4}$. If it is negative, one may obtain a non-unitary $\mathrm{CFT}_{4}$. In this sense, for $\hat{r}>1 / \sqrt{2}, 5 \mathrm{DTBH}$ provides a nonunitary conformal field theory because of $E_{C F T}^{t}>0$ and $E_{c}^{t}<0$. Similarly, for TGBAdS with $\hat{c}<0$ and $\hat{r}>1 / \sqrt{2}$, it provides a non-unitary conformal field theory with $E_{C F T}^{g}>0$ and $E_{c}^{g}<0$. For $\hat{r}<1 / \sqrt{2}$, two cases are not well defined because of negative energy and temperature on the boundary. Hence we do not have any Cardy-Verlinde formula for the 5DTBH and TGBAdS.

Finally, we introduce the Cardy-Verlinde formula (54) to show whether or not the substraction scheme does improve the boundary $\mathrm{CFT}_{4}$ of the topological black hole. However, it does not resolve an original issue of the non-existence of Cardy-Verlinde formula for the topological black holes. 


\section{Discussion}

We believe that for a large class of black holes, the Bekenstein-Hawking entropy receives logarithmic corrections due to thermodynamic fluctuations around thermal equilibrium. However, small black holes with hyperbolic horizon have the negative energy, even though they have positive heat capacity. Hence, a direct application of Eq.(1) to these black holes is not suitable for investigating their thermodynamic fluctuations. We resolve this issue by introducing the mass of extremal black hole as the background state energy for 4DTBH, MTZ, 5DTBH, TGBAdS black holes. In order to avoid negative mass problem, we use the substraction scheme. The entropy-corrected formula is not changed because the substraction scheme corresponds to a constant shifting from $M$ to $E=M-M_{e}$. We have used Eq.(11) to study thermal fluctuations of black holes with hyperbolic horizon.

Especially, we find that the MTZ black hole dressed by a scalar has the best thermodynamic property in $\mathrm{AdS}_{4}$ spacetimes as the BTZ black hole does show in $\mathrm{AdS}_{3}$ spacetimes. Hence we propose the MTZ black hole with hyperbolic horizon as the corner stone to study the $4 \mathrm{D}$ black holes in $\mathrm{AdS}_{4}$ spacetimes, better than the Schwarzschild-AdS black hole. This is because the Schwarzschild-AdS black hole with spherical horizon could not provide a completely thermodynamic picture as the MTZ black hole does show [20, 22].

\section{Acknowledgments}

This work was in part supported by the Korea Research Foundation Grant (KRF-2005013-C00018) and the SRC Program of the KOSEF through the Center for Quantum Spacetime (CQUeST) of Sogang University with grant number R11-2005-021-03001-0.

\section{References}

[1] R. K. Kaul and P. Majumdar, Phys. Lett. B 439, 267 (1998) gr-qc/9801080; R. K. Kaul and P. Majumdar, Phy. Rev. Lett. 56, 5255 (2000) gr-qc/0002040]; S. Carlip, Class. Quant. Grav. 17, 4175 (2000) [gr-qc/0005017]; T. R. Govindarajan, R.K. Kaul and V. Suneeta, Class. Quant. Grav. 18, 2877 (2001) gr-qc/0104010; D. Birmingham and S. Sen, Phys. Rev. D 63, 047501 (2001) hep-th/0008051].

[2] S. Das, P. Majumdar and R. K. Bhaduri, Class. Quant. Grav. 19, 2355 (2002) arXiv:hep-th/0111001.

[3] D. J. Gross, M. J. Perry and L. G. Yaffe, Phys. Rev. D 25, 330 (1982). 
[4] J. W. York, Phys. Rev. D 33, 2092 (1986).

[5] S. W. Hawking and D. N. Page, Commun. Math. Phys. 87, 577 (1983).

[6] J. D. Brown, J. Creighton and R. B. Mann, Phys. Rev. D 50, 6394 (1994) gr-qc/9405007.

[7] E. Witten, Adv. Theor. Math. Phys. 2, 505 (1998) hep-th/9803131.

[8] A. Chamblin, R. Emparan, C. V. Johnson and R. C. Myers, Phys. Rev. D 60, 064018 (1999). arXiv:hep-th/9902170.

[9] J. P. S. Lemos, Phys. Lett. B 353, 46 (1995).

[10] L. Vanzo, Phys. Rev. D 56, 6475 (1997).

[11] D. R. Brill, J. Louko and P. Peldan, Phys. Rev. D 56, 3600 (1997).

[12] Y. S. Myung, Phys. Lett. B 645, 369 (2007).

[13] D. Birmingham and S. Mokhtari, Phys. Rev. D 76, 124039 (2007).

[14] D. Birmingham, Class. Quant. Grav. 16, 1197 (1999) hep-th/9808032.

[15] C. Martinez, R. Troncoso and J. Zanelli, Phys. Rev. D 70, 084035 (2004).

[16] G. Koutsoumbas, S. Musiri, E. Papantonopoulos and G. Siopsis, JHEP 0610, 006 (2006) arXiv:hep-th/0606096.

[17] Y. S. Myung, Phys. Lett. B 663, 111 (2008) [arXiv:0801.2434 [hep-th]].

[18] M. Nadalini, L. Vanzo and S. Zerbini, Phys. Rev. D 77, 024047 (2008) arXiv:0710.2474 [hep-th]].

[19] S. Mukherji and S. S. Pal, JHEP 0205, 026 (2002) |hep-th/0205164.

[20] L. Cappiello and W. Muck, Phys. Lett. B 522, 139 (2001) hep-th/0107238.

[21] R. G. Cai, Phys. Rev. D 63, 124018 (2001) hep-th/0102113.

[22] Y. S. Myung, Phys. Lett. B 574, 289 (2003) hep-th/0308191.

[23] R. G. Cai, Phys. Rev. D 65, 084014 (2002) arXiv:hep-th/0109133. 
[24] Y. M. Cho and I. P. Neupane, Phys. Rev. D 66, 024044 (2002) arXiv:hep-th/0202140.

[25] Y. S. Myung, Y. W. Kim and Y. J. Park, Eur. Phys. J. C 58, 337 (2008) arXiv:0806.4452 [gr-qc]].

[26] E. Verlinde, hep-th/0008140.

[27] E. Witten, Adv. Theor. Math. Phys. 2, 505 (1998) arXiv:hep-th/9803131].

[28] M. R. Setare, Eur. Phys. J. C 33, 555 (2004) arXiv:hep-th/0309134].

[29] Y. S. Myung, Class. Quant. Grav. 21, 1279 (2004) hep-th/0309179]. 